VoL. 47 (1993) [443-455]

\title{
THE APPROXIMATE SUBDIFFERENTIAL OF COMPOSITE FUNCTIONS
}

\section{A. Jourani and L. Thibault}

This paper deals with the approximate subdifferential chain rule in a Banach space. It establishes specific results when the real-valued function is locally Lipschitzian and the mapping is strongly compactly Lipschitzian.

\section{IntRoduction}

In [8] we have proved that, under the metric regularity assumption (a general constraint qualification), a point $\bar{x}$ is a local minimum to the constrained problem

$$
\text { minimise } g(x) \quad \text { subject to } x \in C \text { and } G(x) \in D
$$

(where $g: X \rightarrow \mathbb{R}$ and $G: X \rightarrow Y$ are locally Lipschitz at $\bar{x}$ and $C$ and $D$ are two closed subsets of the Banach spaces $X$ and $Y$ respectively) if and only if $\bar{x}$ is a local minimum to the unconstrained problem

$$
\text { minimise } f \circ F(x) \quad \text { over all } x \in X
$$

where $F(x)=(g(x), G(x), k d(x ; C)) \in \mathbb{R} \times Y \times \mathbb{R}$ and $f(s, y, t)=s+k d(y, D)+t$. Obviously $f$ and $F$ are also locally Lipschitz. When $Y$ is finite dimensional Clarke's formula says that, for $z:=F(\bar{x})$,

$$
\partial_{c}(f \circ F)(\bar{x}) \subset \overline{c o}\left(\bigcup_{z^{*} \in \partial_{c} f(z)} \partial_{c}\left(z^{*} \circ F\right)(\bar{x})\right)
$$

and hence, because of the convex closure operation $\overline{c o}$, one cannot get directly Lagrange multipliers for problem (P) by applying formula (1) and the well known principle $0 \in$ $\partial_{c}(f \circ F)(\bar{x})$. One of the most important properties of the approximate subdifferential introduced by Mordukhovich [9] is that it satisfies formula (1) without the convex closure operation, that is

$$
\partial_{A}(f \circ F)(\bar{x}) \subseteq \bigcup_{z^{*} \in \partial_{A} f(z)} \partial_{A}\left(z^{*} \circ F\right)(\bar{x})
$$

Received 28 May 1992

Copyright Clearance Centre, Inc. Serial-fee code: 0004-9729/93 \$A2.00+0.00. 
whenever $X$ and $Y$ are both finite dimensional (see $[3,9$, and 7$]$ ) and hence by using our reduction procedure above we can easily derive Lagrange multipliers (relative to the approximate subdifferential) for problem $(\mathcal{P})$ by writing $0 \in \partial_{A}(f \circ F)(\bar{x})$. Moreover these multipliers are also multipliers relative to the Clarke subdifferential since the approximate subdifferential for any locally Lipschitz function is included in the Clarke subdifferential.

Ioffe [6] has extended formula (2) to the case where $X$ and $Y$ are general Banach spaces and $F$ admit a strict prederivative with compact values. Our aim in this paper is to prove (when $X$ and $Y$ are Banach spaces) formula (2) for the larger class of strongly compactly Lipschitzian mappings $F$, a variant of the class of compactly Lipschitzian mappings introduced by the second author in [10]. Many results of this article are largely inspired by the papers [2] and [6] of Ioffe. Because of the importance, in our opinion, of this composition formula, and in order to make the paper self-contained we recall all the notion that we use and we give detailed proofs of the main results.

\section{Preliminaries}

Throughout the paper $X$ and $Y$ are Banach spaces and we denote by $B_{X}, B_{Y}$, $B_{X}^{*}$ and $B_{Y}^{*}$ the closed unit balls of $X, Y, X^{*}$ and $Y^{*}$ respectively and $B(v, s)=$ $\{z:\|v-z\| \leqslant s\}$. By $\langle. ;$.$\rangle we denote the canonical pairing between the space and its$ dual and also the inner product in any Euclidean subspace $L \subseteq X$. We also write

$$
L^{\perp}=\left\{x^{*} \in X^{*}:\left\langle x^{*} ; x\right\rangle=0, \quad \forall x \in L\right\}
$$

If $f$ is an extended-real-valued function on $X$, we write for any subset $D$ of $X$

$$
f_{D}(x)= \begin{cases}f(x), & \text { if } x \in D \\ +\infty, & \text { otherwise }\end{cases}
$$

The function

$$
d^{-} f(x ; h)=\liminf _{\substack{u \rightarrow h \\ t \downarrow 0}} t^{-1}(f(x+t u)-f(x))
$$

is the lower Dini derivative of $f$ at $x$ and

and

$$
\partial^{-} f(x)=\left\{x^{*} \in X^{*}:\left\langle x^{*} ; h\right\rangle \leqslant d^{-} f(x ; h), \quad \forall h \in X\right\}
$$

$$
\partial_{e}^{-} f(x)=\left\{x^{*} \in X^{*}:\left\langle x^{*} ; h\right\rangle \leqslant d^{-} f(x ; h)+\varepsilon\|h\|, \quad \forall h \in X\right\}
$$

are the Dini subdifferential and the Dini $\varepsilon$-subdifferential of $f$ at $x$. 
REMARK. For any locally Lipschitz function $f$ one has

$$
d^{-} f(x ; h)=\liminf _{t \downarrow 0} t^{-1}(f(x+t h)-f(x)) .
$$

Definition 1.1: A collection $\mathcal{L}$ of closed subspaces of $X$ will be called admissible if

(a) every $x \in X$ belongs to some $L \in \mathcal{L}$;

(b) for any $L_{1}, L_{2} \in \mathcal{L}$ there is an $L \in \mathcal{L}$ containing both $L_{1}$ and $L_{2}$.

EXAMPLE: The family $\mathcal{F}$ of all finite dimensional subspaces of $X$ is an admissible one.

that is

In all the sequel $\lim \sup \partial^{-} f_{x+L}(x)$ will denote the weak-star superior limit set,

$$
\underset{x \stackrel{f}{\rightarrow}}{\limsup } \partial^{-} f_{x+L}(x)=\left\{x^{*} \in X^{*}: x^{*}=w^{*}-\lim _{i} x_{i}^{*}, x_{i}^{*} \in \partial^{-} f_{x_{i}+L}(x) \text { and } x_{i} \stackrel{f}{\longrightarrow} \bar{x}\right\}
$$

where $x \stackrel{f}{\longrightarrow} \bar{x}$ means that $x \rightarrow \bar{x}$ and $f(x) \rightarrow f(\bar{x})$.

Definition 1.2: [4] Let $\mathcal{F}$ be the previous collection and $f$ be a lower semicontinuous function on $X$ with $|f(\bar{x})|<+\infty$. The $A$-approximate subdifferential of $f$ at $\overline{\boldsymbol{x}}$ is defined by

$$
\partial_{A} f(\bar{x})=\bigcap_{L \in \mathcal{F}} \limsup _{x \stackrel{f}{\rightarrow} \bar{x}} \partial^{-} f_{x+L}(x) .
$$

REMARK. The set-valued mapping $x \rightarrow \partial_{A} f(x)$ is upper semicontinuous in the following sense: $\partial_{A} f(x)=\limsup \partial_{A} f(x)$, (see [4]).

$$
\underset{x \stackrel{f}{\rightarrow}}{\vec{x}}
$$

Definition 1.3: [4] One says that $X$ is a weak trustworthy space ( $W T$-space) if for any two lower semicontinuous functions $f^{1}$ and $f^{2}$ on $X$ and any $\varepsilon>0$

$$
\partial_{\varepsilon}^{-}\left(f^{1}+f^{2}\right)(x) \subset \underset{\substack{x_{i} \\ i=1,2}}{\lim \sup _{i}}\left(\partial_{\varepsilon}^{-} f^{1}\left(x_{1}\right)+\partial_{\varepsilon}^{-} f^{2}\left(x_{2}\right)\right) .
$$

EXAMPLE: Every separable space is a $W T$-space (see [5]).

Lemma 1.4. Let $T: X \rightarrow Y$ be a surjective continuous linear operator between two Banach spaces $X$ and $Y$ and let $M: Z \rightrightarrows Y$ be a multifunction with nonempty values where $Z$ is a metric space. Then

$$
T^{-1}\left(\|\|-\limsup _{z \rightarrow \bar{z}} M(z)\right) \subset\|\|-\limsup _{z \rightarrow \bar{z}} T^{-1}(M(z)),
$$


where \|\|$-$ lim sup denotes the strong superior limit set.

Proof: Let $T x \in \limsup _{z \rightarrow \bar{z}} M(z)$. Without loss of generality we may assume that there is $z_{n} \rightarrow \bar{z}$ and $y_{n} \in M\left(z_{n}\right)$ such that $y_{n} \rightarrow T x$. From the surjectivity of $T$ one has (see for example [1]) the existence of $a \geqslant 0$ and $r>0$ such that

$$
d\left(x^{\prime}, T^{-1}\left(y^{\prime}\right)\right) \leqslant \operatorname{ad}\left(y^{\prime}, T x^{\prime}\right)
$$

for all $x^{\prime} \in B(x, r)$ and $y^{\prime} \in B(T x, r)$, where $d(v, D)=\inf \left\{\left\|v-v^{\prime}\right\|: v^{\prime} \in D\right\}$. So there is $n_{0} \in \mathbb{N}$ such that for $n>n_{0}$ one has $y_{n} \in B(T x, r)$ and

$$
d\left(x, T^{-1}\left(y_{n}\right)\right) \leqslant a d\left(y_{n}, T x\right),
$$

and hence there is $x_{n} \in T^{-1}\left(y_{n}\right)$ such that

$$
d\left(x, x_{n}\right) \leqslant 2 a d\left(y_{n}, T x\right)
$$

which implies that $x \in \limsup _{n \rightarrow+\infty} T^{-1}\left(y_{n}\right) \subset \limsup _{z \rightarrow \bar{z}} T^{-1}(M(z))$.

The following lemma and the next propositions will be used in Section 2.

Lemma 1.5. Let $L$ be a finite dimensional subspace of $X, f^{1}$ and $f^{2}$ two lower semi-continuous functions on $X$ and $\delta>0$. Then

$$
\partial_{\varepsilon}^{-}\left(f^{1}+f^{2}\right)_{x+L}(x) \subset \underset{\substack{x_{i} \\ i=1,2}}{\limsup }\left(\partial_{e}^{-} f_{x_{1}+L}^{1}\left(x_{1}\right)+\partial_{e}^{-} f_{x_{2}+L}^{2}\left(x_{2}\right)+L^{\perp}\right) .
$$

Proof: Let $P: L \rightarrow X$ be the imbedding operator and let $P^{*}: X^{*} \rightarrow X^{*} / L^{\perp}$ be the canonical projection. For each $h \in L$ we set $g_{1}(h)=f_{x+L}^{1}(x+P h)$ and $g_{2}(h)=$ $f_{x+L}^{2}(x+P h)$. It is not difficult to see that for any $u_{1}, u_{2} \in L$

$d^{-} g_{1}\left(u_{1}, h\right)=d^{-} f_{x+P u_{1}+L}^{1}\left(x+P u_{1}, P h\right)$ and $d^{-} g_{2}\left(u_{2}, h\right)=d^{-} f_{x+P u_{2}+L}^{2}\left(x+P u_{2}, P h\right)$.

Let us note that $\partial_{\varepsilon}^{-} f_{x+P u_{1}+L}^{1}\left(x+P u_{1}\right)=\left(P^{*}\right)^{-1}\left(\partial_{e}^{-} g_{1}\left(u_{1}\right)\right)$ since for $P^{*} x \in \partial_{e}^{-} g_{1}\left(u_{1}\right)$ we have for all $h \in L$

$$
\begin{aligned}
\left\langle P^{*} x^{*}, h\right\rangle & \leqslant d^{-} g_{1}\left(u_{1}, h\right)+\varepsilon\|h\| \\
& =d^{-} f_{x+P u_{1}+L}^{1}\left(x+P u_{1}, P h\right)+\varepsilon\|h\|
\end{aligned}
$$

which is equivalent to $x^{*} \in \partial_{e}^{-} f_{x+P u_{1}+L}^{1}(x+P u)$. So as $L$ is a $W T$-space

$$
\partial_{\varepsilon}^{-}\left(g_{1}+g_{2}\right)(0) \subset \underset{\substack{u_{i}=g_{i}, 0 \\ i=1,2}}{\limsup }\left(\partial_{\varepsilon}^{-} g_{1}\left(u_{1}\right)+\partial_{\varepsilon}^{-} g_{2}\left(u_{2}\right)\right)
$$


and hence becasue the surjectivity of $P^{*}$ and Lemma 1.4 it follows that

$$
\begin{aligned}
& \partial_{\varepsilon}^{-}\left(f^{1}+f^{2}\right)_{x+L}(x)=\left(P^{*}\right)^{-1}\left(\partial_{\varepsilon}^{-}\left(g_{1}+g_{2}\right)(0)\right) \\
& \subset\left(P^{*}\right)^{-1}\left(\|\|-\underset{\substack{u_{2} g_{i} \rightarrow 0 \\
i=1,2}}{\limsup }\left(\partial_{\varepsilon}^{-} g_{1}\left(u_{1}\right)+\partial_{\varepsilon}^{-} g_{2}\left(u_{2}\right)\right)\right) \\
& C\|\|-\lim \sup \left(P^{*}\right)^{-1}\left(\partial_{\varepsilon}^{-} g_{1}\left(u_{1}\right)+\partial_{\varepsilon}^{-} g_{2}\left(u_{2}\right)\right) \\
& \underset{i=1,2}{\substack{\boldsymbol{i}_{i} \\
i=1,2}} \\
& \subset \lim \sup \left(P^{*}\right)^{-1}\left(\partial_{\varepsilon}^{-} g_{1}\left(u_{1}\right)+\partial_{\varepsilon}^{-} g_{2}\left(u_{2}\right)\right) \\
& \underset{i=1,2}{u_{i}} \underset{g_{2}}{\stackrel{g^{2}}{0}} \\
& C \lim \sup \left[\left(P^{*}\right)^{-1}\left(\partial_{\varepsilon}^{-} g_{1}\left(u_{1}\right)\right)+\left(P^{*}\right)^{-1}\left(\partial_{\varepsilon}^{-} g_{2}\left(u_{2}\right)\right)+L^{\perp}\right] \\
& \underset{i=1,2}{u_{i} \underset{g_{i}}{g_{i}}} \mathbf{0} \\
& C \lim \sup \left[\partial_{e}^{-} f_{x+P u_{1}+L}^{1}\left(x+P u_{1}\right)+\partial_{e}^{-} f_{x+P u_{2}+L}^{2}\left(x+P u_{2}\right)+L^{\perp}\right] \\
& \underset{i=1,2}{u_{i} \underset{g_{\mathbf{m}}}{\longrightarrow} 0} \\
& C \lim \sup \left[\partial_{e}^{-} f_{x_{1}+L}^{1}\left(x_{1}\right)+\partial_{e}^{-} f_{x_{2}+L}^{2}\left(x_{2}\right)+L^{\perp}\right] . \\
& \underset{x_{i}}{\stackrel{f_{i}}{f^{i}} \boldsymbol{x}} \boldsymbol{x}
\end{aligned}
$$

In the sequel we shall denote by $d(S ;$.$) the distance function to a subset S$ of $X$. The notation $x \stackrel{S}{\longrightarrow} \bar{x}$ will mean $x \longrightarrow \bar{x}$ and $x \in S$.

Proposition 1.6. [4] Let $\mathcal{L}$ be an admissible collection of $W T$-subspaces of $X$. Then

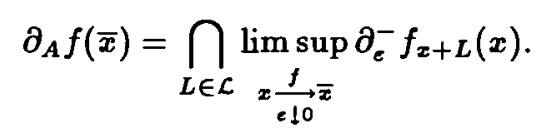

Moreover if $S$ is a subset of $X$ which is closed around $\bar{x} \in S$ (that is $S \cap B(\bar{x}, r)$ is closed for some closed ball $B(\bar{x}, r)$ ), then

$$
\partial_{A} d(S ; \bar{x})=\bigcap_{L \in \mathcal{C}} \limsup _{\substack{x \in s^{s} \bar{x} \\ \varepsilon \downarrow 0}} \partial_{\varepsilon}^{-} d_{x+L}(S ; x) .
$$

Remark. Following Ioffe [2] we see that for any $\varepsilon>0$ and any $L \in \mathcal{L}$, each $x^{*} \in$ $\partial_{e}^{-} d_{x+L}(S ; x)$ satisfies $\left\langle x^{*}, h\right\rangle \leqslant(1+\varepsilon)\|h\|$ for all $h \in L$. Therefore $x^{*} \in(1+\varepsilon) B_{x^{*}}+$ $L^{\perp}$ (where $B_{x^{*}}$ is the closed unit ball of $X^{*}$ ) and hence

$$
\partial_{e}^{-} d_{x+L}(S ; x) \subset \partial_{e}^{-} d_{x+L}(S ; x) \cap(1+\varepsilon) B_{X}^{*}+L^{\perp} .
$$


As the reverse inclusion is obvious we obtain

$$
\partial_{e}^{-} d_{x+L}(S ; x)=\partial_{e}^{-} d_{x+L}(S ; x) \cap(1+\varepsilon) B_{X}^{*}+L^{\perp}
$$

which ensures that

$$
\partial_{A} d(S ; \bar{x})=\bigcap_{L \in \mathcal{L}} \limsup _{x \underset{\varepsilon \downarrow 0}{s} \bar{x}} \partial_{\varepsilon}^{-} d_{x+L}(S ; x) \cap(1+\varepsilon) B_{X}^{*}
$$

Theorem 1.7. [4] Let $f$ be a lower semicontinuous function on $X$ with $|f(\bar{x})|<+\infty$ and let $g$ be a Lipschitz function at $\bar{x}$. Then

$$
\partial_{A}(f+g)(\bar{x}) \subset \partial_{A} f(\bar{x})+\partial_{A} g(\bar{x})
$$

Proposition 1.8. [12] Let $F$ be a mapping from $X$ into $Y$ which is Lipschitz at $\bar{x}$. Then

$$
\|y-F(x)\| \leqslant(k+1) d(G r F ; x, y)
$$

for all $x$ and $y$ belonging to some neighbourhood of $\bar{x}$ and $F(\bar{x})$ respectively, where $k$ is a Lipschitz constant of $F$ at $\bar{x}$ and $G r F$ denotes the graph of $F$, that is $G r F=$ $\{(x, y) \in X \times Y: y=F(x)\}$.

\section{THE MAIN RESULT}

Definition 2.1: [8] A mapping $F: X \rightarrow Y$ is said to be strongly compactly Lipschitzian at $\bar{x}$ if there exists a multifunction $R: X \rightrightarrows \operatorname{Comp}(Y)$, where $\operatorname{Comp}(Y)$ is the collection of all non void \|\| -compact subsets of $Y$, and a function $r: X \times X \rightarrow \mathbb{R}_{+}$ satisfying the following properties:

(1) $\lim _{\substack{x \rightarrow \bar{x} \\ h \rightarrow 0}} r(x, h)=0$,

(2) there is $\mu>0$ such that for all $h \in \mu B_{X}, x \in B(\bar{x} ; \mu)$ and all $\left.t \in\right] 0, \mu[$

$$
t^{-1}(F(x+t h)-F(x)) \in R(h)+\|h\| r(x, t h) B_{X},
$$

(3) $R(0)=\{0\}$ and $R$ is upper semicontinuous.

\section{REMARKS.}

(1) If $F$ is strictly differentiable at $\bar{x}$, then $F$ is strongly compactly Lipschitzian at $\bar{x}$.

(2) If $Y$ is finite dimensional, then $F$ is strongly compactly Lipschitzian at $\bar{x}$ if and only if it is Lipschitzian at $\bar{x}$. 
Let us recall some results concerning these mappings. The proof of the following is similar to the one established by Thibault [11].

Proposition 2.2.

(1) Every strongly compactly Lipschitzian mapping at $\bar{x}$ is Lipschitzian at $\overline{\boldsymbol{x}}$.

(2) The sum of two strongly compactly Lipschitzian mappings is strongly compactly Lipschitzian.

The proof of the following result is inspired by Ioffe [2].

Proposit TION 2.3. Let $F: X \rightarrow Y$ be a strongly compactly Lipschitzian mapping at $\bar{x}, c>0$ and $\varepsilon>0$. Then there is $\gamma>0$ such that for all $L \in \mathcal{F}$ there exists a $\omega^{*}$-neighbourhood $U$ of 0 in $Y^{*}$ such that for all $x \in B(\bar{x} ; \gamma), v^{*} \in U$ with $\left\|v^{*}\right\| \leqslant c$, $\sigma>0$ and all $x^{*} \in \partial_{\sigma}^{-}\left(v^{*} \circ F\right)_{x+L}(x)$ one has

$$
x^{*} \in(2 \sigma+\varepsilon) B_{X}^{*}+L^{\perp} .
$$

Proof: Since $F$ is strongly compactly Lipschitzian at $\bar{x}$ there are $\mu>0$, a multifunction $R: X \rightrightarrows \operatorname{Comp}(Y)$ and a function $r: X \times X \rightarrow \mathbb{R}_{+}$satisfying the conditions (1), (2) and (3) of Definition 2.1. Let $\varepsilon>0$ and $c>0$ be given. The compactness of the closed unit ball $B_{L}$ of $L$ in $(X ;\|\|)$ ensures the existence of elements $h_{1}, \ldots, h_{p}$ of $B_{L}$ such that

$$
B_{L} \subset \bigcup_{j=1}^{p}\left(h_{j}+\frac{\varepsilon}{2(c k+\varepsilon)} B_{L}\right)
$$

where $k$ is a Lipschitz constant of $F$ at $\bar{x}$. The compactness of $R\left(\mu h_{j}\right)$, for $j=$ $1, \ldots, p$, in $(Y ;\|\|)$ also ensures the existence of $v_{1}, \ldots, v_{q_{j}}$ in $R\left(\mu h_{j}\right)$ such that

$$
R\left(\mu h_{j}\right) \subset \bigcup_{i=1}^{q_{j}}\left(v_{i}+\frac{\mu \varepsilon}{8 c} B_{Y}\right) .
$$

For each $j=1, \ldots, p$ put $U_{j}=H_{j}^{\perp}+(\mu \varepsilon) /(8 b) B_{Y}^{*}$, where $H_{j}$ is the subspace of $Y$ generated by $\left\{v_{1}, \ldots, v_{q_{j}}\right\}$ and where $b=\max _{j=1, \ldots, p} \sup _{z \in R\left(\mu h_{j}\right)}\|z\|$. Then for each $j=1, \ldots, p U_{j}$ is a $w^{*}$-neighbourhood of 0 in $Y^{*}$ and for all $v^{*} \in U_{j}$, with $\left\|v^{*}\right\| \leqslant c$, and $z \in R\left(\mu h_{j}\right)$

$$
\left\langle v^{*} ; z\right\rangle \leqslant \frac{\mu \varepsilon}{4}
$$


If we take $U=\bigcap_{j=1}^{p} U_{j}$ then $U$ is a $w^{*}$-neighbourhood of 0 and satisfies relation (2.2) for all $v^{*} \in U$ with $\left\|v^{*}\right\| \leqslant c$ and $z \in \bigcup_{j=1}^{p} R\left(\mu h_{j}\right)$. Because of (1) of Definition 2.1 if we put $r(x)=\underset{h \rightarrow 0}{\limsup } r(x, h)$ one can get $\gamma \in] 0, \mu[$ such that for all $x \in B(\bar{x} ; \gamma)$

$$
\operatorname{cr}(x) \leqslant \frac{\varepsilon}{4}
$$

Let $x \in B(\bar{x} ; \gamma), \sigma>0$ and $v^{*} \in U$ with $\left\|v^{*}\right\| \leqslant c$ be fixed and let $x^{*} \in$ $\partial_{\sigma}^{-}\left(v^{*} \circ F\right)_{x+L}(x)$. Then for all $j=1, \ldots, p$

$$
\begin{aligned}
\left\langle x^{*} ; \mu h_{j}\right\rangle & \leqslant d^{-}\left(v^{*} \circ F\right)_{x+L}\left(x ; \mu h_{j}\right)+\mu \sigma\left\|h_{j}\right\| \\
& \leqslant \liminf _{t \downarrow 0} t^{-1}\left\langle v^{*} ; F\left(x+t \mu h_{j}\right)-F(x)\right\rangle+\mu \sigma
\end{aligned}
$$

because $\left\|h_{j}\right\| \leqslant 1$. As $x \in B(\bar{x} ; \gamma) \subset B(\bar{x} ; \mu)$ it follows that for each $j=1, \ldots, p$

$$
\left\langle x^{*} ; \mu h_{j}\right\rangle \leqslant \sup _{z \in R(\mu h,)}\left\langle v^{*} ; z\right\rangle+\mu r(x)\left\|v^{*}\right\|+\mu \sigma
$$

and hence by (2.2) and (2.3)

$$
\left\langle x^{*} ; \mu h_{j}\right\rangle \leqslant \frac{\mu \varepsilon}{4}+\frac{\mu \varepsilon}{4}+\mu \sigma
$$

which implies that

$$
\left\langle x^{*} ; h_{j}\right\rangle \leqslant \frac{\varepsilon}{2}+\sigma
$$

But for any $h \in B_{L}$ where exists, by (2.1), some $j \in\{1, \ldots, p\}$ such that

$$
\left\|h-h_{j}\right\| \leqslant \frac{\varepsilon}{2(c k+\varepsilon)}
$$

which together with relation (2.4) implies that

$$
\begin{aligned}
\left\langle x^{*} ; h\right\rangle & =\left\langle x^{*} ; h-h_{j}\right\rangle+\left\langle x^{*} ; h_{j}\right\rangle \\
& \leqslant \underline{d}\left(v^{*} \circ F\right)_{x+L}\left(x ; h-h_{j}\right)+\sigma\left\|h-h_{j}\right\|+\frac{\varepsilon}{2}+\sigma \\
& \leqslant c k\left\|h-h_{j}\right\|+\sigma\left\|h-h_{j}\right\|+\frac{\varepsilon}{2}+\sigma \\
& \leqslant \frac{\varepsilon}{2}+\sigma+\frac{\varepsilon}{2}+\sigma \\
& =\varepsilon+2 \sigma .
\end{aligned}
$$


By the homogeneity of this it follows that for all $h \in L$

$$
\left\langle x^{*} ; h\right\rangle \leqslant(\varepsilon+2 \sigma)\|h\|
$$

and hence $x^{*} \in(\varepsilon+2 \sigma) B_{X}^{*}+L^{\perp}$.

In the sequel $\mathcal{F}_{X}$ and $\mathcal{F}_{Y}$ denote the families of all finite dimensional subspaces of $X$ and $Y$ respectively.

Some techniques in the proof of the following proposition come from Ioffe [6].

Proposition 2.4. Let $F: X \rightarrow Y$ be a strongly compactly Lipschitzian mapping at $\bar{x}$ and let $k$ be a Lipschitz constant of $F$ over $\bar{x}+\delta B_{x}$ and $\bar{y}=F(\bar{x})$. Then the following assertions are equivalent:

(i) $\left(x^{*},-y^{*}\right) \in(k+1)\left\|y^{*}\right\| \partial_{A} d(G r F ; \bar{x}, \bar{y})$

(ii) $\left(x^{*},-y^{*}\right) \in \mathbb{R}_{+} \partial_{A} d(G r F ; \bar{x}, \bar{y})$

(iii) $x^{*} \in \partial_{A}\left(y^{*} \circ F\right)(\bar{x})$.

Proof: Since $F$ is strongly compactly Lipschitzian at $\bar{x}$, there are a multifunction $R: X \rightrightarrows \operatorname{Comp}(Y)$, a function $x: X \times X \rightarrow \mathbb{R}_{+}$with $\lim _{\substack{x \rightarrow \bar{x} \\ h^{\prime} \rightarrow 0}} r\left(x, h^{\prime}\right)=0$ and $s>0$ such that for all $x \in B(x, s), t \in] 0, s\left[\right.$ and $h \in B_{X}$

$$
t^{-1}(F(x+t(s h))-F(x)) \in R(s h)+s\|h\| r(x, t(s h)) B_{Y} .
$$

Let $L \in \mathcal{F}_{X}$. Then the closed unit ball $B_{L}$ of $L$ is a compact subset of $(X,\|\|)$ and from the upper semicontinuity property of $R$ the set $R\left(s B_{L}\right)$ is a compact subset of $(Y,\|\|)$. Put $V_{L}=c l_{Y}\left[\operatorname{vect}\left(R\left(s B_{L}\right)\right)\right]$. Then $V_{L}$ is a separable subspace of $Y$ and for all $M \in \mathcal{F}_{Y}$ the subspace $\overline{M+V}{ }_{L}=c l_{Y}\left[M+V_{L}\right]$ of $Y$ is also separable and hence the family $\left\{L \times \overline{M+V}_{L}\right\}_{L \times M \in \mathcal{F}_{X} \times \mathcal{F}_{Y}}$ is an admissible family of $W T$-subspaces of $X \times Y$ (see [5] and the example following Definition 1.3).

(1) (i) $\rightarrow$ (ii): this implication is obvious.

(2) (ii) $\rightarrow$ (iii): let $\left(x^{*},-y^{*}\right) \in \mathbb{R}_{+} \partial_{A} d(G r F ; \bar{x}, \bar{y})$. Then there exists $\left(u^{*},-v^{*}\right) \in$ $\partial_{A} d(G r F ; \bar{x}, \bar{y})$ such that $\left(x^{*},-y^{*}\right)=\lambda\left(u^{*},-v^{*}\right)$, with $\lambda \geqslant 0$. Then by the remark following Proposition 1.6 for each $L \in \mathcal{F}_{X}$ and each $M \in \mathcal{F}_{Y}$ there are nets $\varepsilon_{i} \downarrow 0$ with $\varepsilon_{i}<1, x_{i} \rightarrow \bar{x}$ and $\left(u_{i}^{*}, v_{i}^{*}\right) \stackrel{w^{*}}{\longrightarrow}\left(u^{*}, v^{*}\right)$ such that

$$
\left(u_{i}^{*},-v_{i}^{*}\right) \in\left(\partial_{\varepsilon_{i}}^{-} d_{\left(x_{i}, F\left(x_{i}\right)\right)+L x \overline{M+V_{L}}}\left(G r F ; x_{i}, F\left(x_{i}\right)\right)\right) \cap\left(1+\varepsilon_{i}\right) B_{X}^{*} .
$$

For any fixed $\varepsilon>0$ there exists $i_{0}$ and $\alpha>0$ such that $x_{i} \in \bar{x}+(\delta / 2) B_{X}$ and $r\left(x_{i}, h^{\prime}\right)<1 / 2 \varepsilon$ for all $i>i_{0}$ and $\left\|h^{\prime}\right\| \leqslant \alpha$, since $\lim _{\substack{x \rightarrow \bar{x} \\ h^{\prime} \rightarrow 0}} r\left(x, h^{\prime}\right)=0$. Let $i>i_{0}$ be $\|h\|=1$ and let $t_{n} \downarrow 0$ be such that

$$
t_{n}^{-1}\left\langle v_{i}^{*} ; F\left(x_{i}+t_{n}(s h)\right)-F\left(x_{i}\right)\right\rangle \rightarrow d^{-}\left(v_{i}^{*} \circ F\right)_{x_{i}+L}\left(x_{i} ; s h\right) .
$$


From (2.4.1) there are $a_{n} \in R(s h) \subset V_{L}$ and $b_{n} \in s r\left(x_{i}, t_{n}(s h)\right) B_{Y}$ such that

$$
t_{n}^{-1}\left(F\left(x_{i}+t_{n}(s h)\right)-F\left(x_{i}\right)\right)=a_{n}+b_{n} .
$$

Note that $\left\|b_{n}\right\|<s \varepsilon$ for $n$ large enough. As $R(s h)$ is a compact set we may assume that $a_{n} \rightarrow a \in V_{L}$. Thus

$$
\begin{aligned}
\left\langle u_{i}^{*} ; s h\right\rangle & \leqslant\left\langle v_{i}^{*} ; a\right\rangle+\varepsilon_{i}(\|s h\|+\|a\|)+\liminf _{t \perp 0} t^{-1} d\left(G r F ; x_{i}+t s h, F\left(x_{i}\right)+t a\right) \\
& \leqslant\left\langle v_{i}^{*} ; a\right\rangle+\varepsilon_{i}(\|s h\|+\|a\|)+\liminf _{t \rightarrow+\infty} f_{n}^{-1} d\left(G r F ; x_{i}+t_{n} s h, F\left(x_{i}\right)+t_{n} a\right) \\
& \leqslant\left\langle v_{i}^{*} ; a\right\rangle+\varepsilon_{i}(\|s h\|+\|a\|)+s \varepsilon
\end{aligned}
$$

because by (2.4.2) $\left(x_{i}+t_{n} s h, F\left(x_{i}\right)+t_{n}\left(a_{n}+b_{n}\right)\right) \in G r F$ and $\left\|b_{n}\right\|<\varepsilon s$. As $a_{n} \rightarrow a$, $\left\|b_{n}\right\|<s \varepsilon,\left\|v_{i}^{*}\right\| \leqslant 2,\|a\| \leqslant(k+\varepsilon) s$ and $\lim _{n \rightarrow \infty}\left\langle v_{i}^{*} ; a_{n}+b_{n}\right\rangle=d^{-}\left(v_{i}^{*} \circ F\right)_{x_{i}+L}\left(x_{i}, s h\right)$ one has

$$
\left\langle u_{i}^{*} ; h\right\rangle \leqslant d^{-}\left(v_{i}^{*} \circ F\right)_{x_{i}+L}\left(x_{i} ; h\right)+\varepsilon_{i}(k+1+\varepsilon)+3 \varepsilon .
$$

Thus for $E(\varepsilon, i)=\varepsilon_{i}(k+1+\varepsilon)+3 \varepsilon$ one has

$$
u_{i}^{*} \in \partial_{E(e, i)}^{-}\left(v_{i}^{*} \circ F\right)_{x_{i}+L}\left(x_{i}\right)
$$

If we write $v_{i}^{*} \circ F=\left(v_{i}^{*}-v^{*}\right) \circ F+v^{*} \circ F$ we can get, by Lemma 1.5, some nets $u_{i} \rightarrow \bar{x}$, $v_{i} \rightarrow \bar{x}, z_{i}^{*} \in \partial_{E(e, i)}^{-}\left(v^{*} \circ F\right)_{u_{i}+L}\left(u_{i}\right)$ and $q_{i}^{*} \in\left[\partial_{E(e, i)}^{-}\left(\left(v_{i}^{*}-v^{*}\right) \circ F\right)_{v_{i}+L}\left(v_{i}\right)+L^{\perp}\right]$ such that $z_{i}^{*}+q_{i}^{*} \stackrel{w^{*}}{\longrightarrow} u^{*}$. But $v_{i}^{*}-v^{*} \stackrel{w^{*}}{\longrightarrow} O$ and $\left(v_{i}^{*}-v^{*}\right)_{i}$ is bounded and hence by Proposition 2.3 one has the existence of $i(\varepsilon)>i_{0}$ such that $q_{i}^{*} \in(\varepsilon+2 E(\varepsilon, i)) B_{Y}^{*}+L^{\perp}$ for all $i>i(\varepsilon)$. Thus

$$
\begin{aligned}
& u^{*} \in \underset{\substack{i \\
\varepsilon \downarrow 0}}{\lim \sup }\left(\partial_{E(e, i)}^{-}\left(v^{*} \circ F\right)_{u_{i}+L}\left(u_{i}\right)+(\varepsilon+2 E(\varepsilon, i)) B_{X}^{*}+L^{\perp}\right)
\end{aligned}
$$

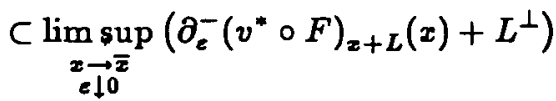

and hence

$$
\begin{aligned}
& u^{*} \in \bigcap_{L \in \mathcal{F}_{X}} \limsup _{\substack{x \rightarrow \bar{x} \\
e \downarrow 0}}\left(\partial_{\varepsilon}^{-}\left(v^{*} \circ F\right)_{x+L}(x)+L^{\perp}\right) \\
& =\limsup _{\substack{x \rightarrow \bar{x} \\
c \backslash 0 \\
L \in \mathcal{F}_{X}}}\left(\partial_{\varepsilon}^{-}\left(v^{*} \circ F\right)_{x+L}(x)+L^{\perp}\right)
\end{aligned}
$$

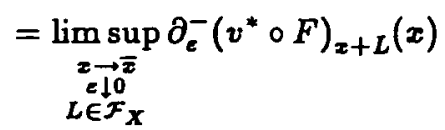

$$
\begin{aligned}
& =\partial_{A}\left(v^{*} \circ F\right)(\bar{x}) \text {. }
\end{aligned}
$$


It follows that $x^{*} \in \lambda \partial_{A}\left(v^{*} \circ F\right)(\bar{x})=\partial_{A}\left(\lambda v^{*} \circ F\right)(\bar{x})=\partial_{A}\left(y^{*} \circ F\right)(\bar{x})$.

(3) (iii) $\rightarrow$ (i): Let $x^{*} \in \partial_{A}\left(y^{*} \circ F\right)(\bar{x})$. Then for each $L \in \mathcal{F}_{X}$ there are nets $x_{i} \rightarrow \bar{x}, \varepsilon_{i} \downarrow 0$ and $x_{i}^{*} \stackrel{w^{*}}{\longrightarrow} x^{*}$ such that $x_{i}^{*} \in \partial_{\varepsilon_{i}}^{-}\left(y^{*} \circ F\right)_{x_{i}+L}\left(x_{i}\right)$ which means that for all $h \in L$

$$
\begin{aligned}
\left\langle x_{i}^{*} ; h\right\rangle & \leqslant \underset{\substack{\liminf ^{\prime}{ }^{\prime} \rightarrow h \\
h^{\prime} \rightarrow h}}{-1}\left(\left(y^{*} \circ F\right)_{x_{i}+L}\left(x_{i}+t h^{\prime}\right)-\left(y^{*} \circ F\right)_{x_{i}+L}\left(x_{i}\right)\right)+\varepsilon_{i}\|h\| \\
& =\liminf _{t \downarrow 0} t^{-1}\left\langle y^{*} ; F\left(x_{i}+t h\right)-F\left(x_{i}\right)\right\rangle+\varepsilon_{i}\|h\|
\end{aligned}
$$

because $y^{*} \circ F$ is Lipschitz at $\bar{x}$. This and Proposition 1.8 imply that for all $M \in \mathcal{F}_{Y}$, $h \in L$ and $v \in Y$

$$
\left\langle x_{i}^{*} ; h\right\rangle-\left\langle y^{*} ; v\right\rangle \leqslant(k+1)\left\|y^{*}\right\| d^{-} d_{\left(x_{i}, F\left(x_{i}\right)\right)+L \times M}\left(G r F ; x_{i}, F\left(x_{i}\right) ; h, v\right)+\varepsilon_{i}(\|h\|+\|v\|)
$$

which gives by Proposition 1.6 that $\left(x^{*},-y^{*}\right) \in(k+1)\left\|y^{*}\right\| \partial_{A} d(G r F ; \bar{x}, F(\bar{x}))$.

THEOREM 2.5. Let $F: X \rightarrow Y$ be a strongly compactly Lipschitzian mapping at $\bar{x}$ and let $f: Y \rightarrow \overline{\mathbb{R}}$ be a Lipschitz function at $\bar{y}=F(\bar{x})$. Then

$$
\partial_{A}(f \circ F)(\bar{x}) \subset \bigcup_{y^{*} \partial_{A} f(\bar{y})} \partial_{A}\left(y^{*} \circ F\right)(\bar{x}) .
$$

Proof: Since $f$ and $F$ are Lipschitz at $\bar{y}$ and $\bar{x}$ respectively we have (see Propositions 1.8 and 2.2) the existence of $\alpha>0$ such that for all $x \in B(\bar{x}, \alpha)$ and $y \in B(\bar{y}, \alpha)$

$$
f \circ F(x) \leqslant f(y)+k\|y-F(x)\| \quad \text { and } \quad\|y-F(x)\| \leqslant\left(k^{\prime}+1\right) d(G r F ; x, y)
$$

where $k$ and $k^{\prime}$ are the Lipschitz constants of $f$ and $F$ respectively. If we put $s(x, y)=$ $f(y)+k\left(k^{\prime}+1\right) d(G r F ; x, y)$, we note that for all $x \in B(\bar{x}, \alpha)$ and $y \in B(\bar{y}, \alpha)$

$$
f \circ F(x) \leqslant s(x, y) \quad \text { and } \quad f \circ F(x)=s(x, F(x)) .
$$

For each $(h, v) \in X \times Y$ and for all finite dimensional spaces $L$ and $M$ of $X$ and $Y$ respectively we have

$$
\begin{aligned}
& t^{-1}\left[(f \circ F)_{x+L}(x+t h)-(f \circ F)_{x+L}(x)\right] \\
& \quad \leqslant t^{-1}\left[s_{(x, F(x))+L \times M}(x+t h, F(x)+t v)-s_{(x, F(x))+L \times M}(x, F(x))\right]
\end{aligned}
$$

for all $t$ small enough and $x$ sufficiently close to $\bar{x}$ and hence

$$
d^{-}(f \circ F)_{x+L}(x, h) \leqslant d^{-} s_{(x, F(x))+L \times M}(x, F(x) ; h, v),
$$


which implies that

$$
\partial^{-}(f \circ F)_{x+L}(x) X\{0\} \subset \partial^{-} s_{(x, F(x))+L \times M}(x, F(x)) .
$$

Therefore we obtain that

$$
\begin{aligned}
\partial_{A}(f \circ F)(\bar{x}) X\{0\} & \subset \bigcap_{L, M} \limsup _{x \rightarrow \bar{x}} \partial^{-} s_{(x, F(x))+L \times M}(x, F(x)) \\
& \subset \bigcap_{L, M} \limsup _{(x, y) \rightarrow(\bar{x}, \bar{y})} \partial^{-} s_{(x, y)+L \times M}(x, y)=\partial_{A} s(\bar{x}, \bar{y}) .
\end{aligned}
$$

So Theorem 1.7 ensures that

$$
\partial_{A} f \circ F(\bar{x}) \times\{0\} \subset\{0\} \times \partial_{A} f(\bar{y})+k\left(k^{\prime}+1\right) \partial_{A} d(G r F ; \bar{x}, \bar{y})
$$

and hence it suffices to use Proposition 2.4 to complete the proof.

\section{REFERENCES}

[1] J.M. Borwein, 'Stability and regular pooint of inequality systems', J. Optim. Theory Appl. 48 (1986), 9-52.

[2] A.D. Ioffe, 'Approximate subdifferentials of non-convex functions', Cahier \# 8120 .

[3] A.D. Ioffe, 'Approximate subdifferentials and applications I: The finite dimensional theory', Trans. Amer. Math. Soc. 281 (1984), 389-416.

[4] A.D. Ioffe, 'Approximate subdifferentials and applications II: Functions on locally convex spaces', Mathematika 33 (1986), 111-128.

[5] A.D. Ioffe, 'On subdifferentiability spaces', Ann. New York Acad. Sci. 410 (1983), 107-120.

[6] A.D. Ioffe, 'Approximate subdifferentials and applications III: The metric theory', Mathematika 36 (1989), 1-38.

[7] A. Jourani and L. Thibault, 'The use of metric graphical regularity in approximate subdifferential calculus rules in finite dimensions', Optimization 21 (1990), 509-519.

[8] A. Jourani and L. Thibault, 'A pproximations and metric regularity in mathematical programming in Banach space', Math. Oper. Res. (to appear).

[9] B.S. Mordukhovich, 'Nonsmooth analysis with nonconvex generalized differentials and dual maps', Dokl. Akad. Nauk. USSR 28 (1984), 976-979.

[10] R.T. Rockafellar, 'Extensions of subgradient calculus with applications to optimization', Nonlinear Analysis Th. Meth. Appl. 9 (1985), 665-698.

[11] L. Thibault, 'Subdifferentials of compactly Lipschitzian vector-valued functions', Travaux du séminaire d'analyse convese 8 (1978).

[12] L. Thibault, 'On subdifferentials of optimal value functions', SIAM J. Control Optim. 29 (1991), 1019-1036. 
Université de Bourgogne

Laboratoire d'analyse numérique

B.P. 138

21004 Dijon, Cedex

France
Université de Pau

Laboratoire de Mathématiques appliquées Avenue de l'université

$64000 \mathrm{Pau}$

France 